Check for updates

Cite this: RSC Adv., 2017, 7, 46472

\title{
Enhanced osteointegration of tantalum-modified titanium implants with micro/nano-topography
}

\author{
Junyu Shi, $\uparrow$ Xiaomeng Zhang, $\uparrow$ Shichong Qiao, Jie Ni, Jiaji Mo, Yingxin Gu* \\ and Hongchang Lai $\mathbb{D}$ *
}

Numerous approaches have been used to modify titanium implant surfaces to achieve better osteointegration. In this study, commonly used sandblasted, large grid acid etched (SLA) titanium implants were modified with bioactive trace elements in combination with surface micro/nanotopographical modifications to enhance the osteointegration and antibacterial activity. Briefly, tantalum (Ta) was implanted onto SLA titanium (SLA-Ta) by magnetron sputtering. The modified surface was characterized by scanning electron microscopy (SEM) and X-ray photoelectron spectroscopy (XPS). The electrochemical corrosion properties of two surfaces were measured. The effects of the SLA-Ta surface on rat bone marrow mesenchymal stem cells (rBMSCs), including cell adhesion and proliferation, osteogenic-related gene expression, alkaline phosphatase activity, alizarin red staining, and the immunofluorescence of osteopontin and osteocalcin, were investigated. In addition, the influence of the Wnt/ $\beta$-catenin pathway in this process was explored using western blotting. The SEM and XPS results revealed that $\mathrm{Ta}_{2} \mathrm{O}_{5}$ with micro/nanoscale-topography was established on the titanium surface. $\mathrm{A}$ potentiodynamic polarization study indicated the better corrosion resistance of the modified SLA-Ta surface. Importantly, the SLA-Ta structure not only significantly promoted the initial adhesion activity and proliferation of rBMSCs, but also enhanced osteogenic gene and protein expression, reflecting osteogenic function. Western blot results suggested the possible mechanism of the SLA-Ta surface induced osteogenic differentiation might be the activation of the Wnt/ILK- $\beta$-catenin pathway. These results suggest our developed SLA-Ta surface offers remarkable osteogenic activity and shows great potential for application in orthopaedic and dental implants.

Received 20th July 2017

Accepted 25th September 2017

DOI: $10.1039 / c 7 r a 08036 k$

rsc.li/rsc-advances straightforward surface modification technique that can reinforce these effects simultaneously.

Tantalum (Ta), a potential metallic implant biomaterial, is attracting growing concern owning to its excellent anticorrosion and biocompatibility. Tantalum has regained interest in the biomaterial field mainly due to a new porous (trabecular) tantalum material of micro-porosity, which has been shown to possess excellent osteointegrative properties. ${ }^{6}$ Since then, studies have compared the biocompatibility and osteoconductivity of Ta with that of other common implant materials, such as titanium (Ti), cobalt chromium (CoCr), and hydroxyapatite coatings. ${ }^{7-9} \mathrm{Ta}$ shows remarkable physicochemical stability and excellent biocompatibility compared to that of $\mathrm{Ti}^{8,10}$ And the in vitro study by Sagomonyants et al. also demonstrated that porous Ta even stimulates the proliferation and osteogenesis of osteoblasts when compared with titanium fiber mesh. ${ }^{11}$ Bobyn $e t$ al. used acetabular cups and cylindrical implants in a canine model and found dense bone in-growth into porous Ta. ${ }^{12,13}$ However, the relatively expensive manufacturing costs as well as significantly high elastic modulus unmatched with bone tissue make tantalum unsuitable as medical bulk implant, thus restricting its widespread use in medical practice. ${ }^{14}$
Department of Oral and Maxillo-facial Implantology, Shanghai Ninth People's Hospital, School of Medicine, Shanghai Jiaotong University, 639 Zhizaoju Road, Shanghai 200011, China. E-mail: yingxingu@163.com; lhc9@hotmail.com; Fax: +86 21 53073068; Tel: +862123271699 ext. 5298

$\dagger$ Junyu Shi and Xiaomeng Zhang contributed equally to this work. 
Surface modification is an effective way to adapt the surface properties by introducing the bioactive ions and topographical modification without changing the favorable characteristics of the bulk materials. ${ }^{15}$ Among the numerous modification techniques, magnetron sputtering is a cost-effective, controllable and predictable method which is suitable for biomedical products. ${ }^{16}$ Specific bio-functions can be realized by incorporating certain elements into the biomaterials. For example, a recent study has indicated that the introduction of tantalum onto $\mathrm{TiO}_{2}$ nanotubes significantly strengthens matrix mineralization and osteogenic function in human osteoblasts. ${ }^{17}$ Several studies have demonstrated that the effect of nano-sized topographical features as well as the synergistic effects of micro/ nano-scale hybrid structures on promotion of osteoblast adhesion and activity. ${ }^{18,19}$ In order to achieve the synergistic effects of tantalum and micro/nano topography, we used magnetron sputtering to implant tantalum onto titanium with sandblasted, large-gritted, acid-etched (SLA) surface, which is a commercially available surface for dental implants. However, it remains unclear whether the surface properties altered by magnetron sputtering could induce the corresponding expected biological effects. What's more, the molecular mechanism involved in Tamodified SLA titanium surface inducing osteogenic effect was not understood yet.

In this study, tantalum magnetron sputtering is performed to modify SLA titanium surface to take full advantage of the beneficial properties of SLA surface and tantalum and fabricate SLA-Ta surface with hybrid micro/nano-scale structure. Rat bone marrow mesenchymal stem cells (rBMSCs) were selected to systematically evaluate the osteogenic properties of SLA-Ta surface in vitro. Furthermore, the expression of Wnt/ILK- $\beta$-catenin pathway-related proteins was also measured, trying to explain the possible molecular mechanism of SLA-Ta surface inducing rBMSCs' odontogenic differentiation.

\section{Materials and methods}

\subsection{Preparation and characterization of materials}

Commercial pure titanium discs (diameter: $15 \mathrm{~mm}$, thickness: 1 $\mathrm{mm}$ ) of grade IV with SLA surfaces were acquired according to our previous study. Tantalum was implanted onto SLA titanium disk using magnetron-sputtering technique. ${ }^{20}$ The substrates were sputter-cleaned for $5 \mathrm{~min}$ at a bias of $800 \mathrm{~V}$, duty factor of $30 \%$ and working pressure of $0.02 \mathrm{~Pa}$. Then they were sputtered with Ti for $10 \mathrm{~min}$ at a bias of $250 \mathrm{~V}$, duty factor of $30 \%$ and working pressure of $0.02 \mathrm{~Pa}$. Afterwards, deposition of Ta coatings was conducted at a substrate bias of $150 \mathrm{~V}$, duty factor of $80 \%$, working pressure of $0.02 \mathrm{~Pa}$, and deposition time of $40 \mathrm{~min}$. Both clinic-used materials with different surfaces were provided by Trausim Medical Instrument Co. Ltd (Changzhou, Jiangsu, China).

The field emission scanning electron microscopy (Tokyo, Japan) was used to observe the surface morphologies of two samples and the cross-sectional view of SLA-Ta surface. X-ray photoelectron spectroscopy (Eden Prairie, MN) was utilized to test the chemical compositions of the surfaces. Profilometer (Tokyo, Japan) and goniometer (Contact Angle System JC2000D3, China) were used to assess the surface roughness and the contact angles of two surfaces, respectively. Electrochemical workstation (CHI760c, Shanghai) was used to obtain the dynamic potential polarization curves of two samples as previously published. ${ }^{21}$ Gamma radiation (25KGY) was used to sterilize all samples before experiment.

\subsection{Cell preparation, cell adhesion and cell viability assays}

Rat bone marrow mesenchymal stem cells (rBMSCs) were extracted as we previously illustrated. ${ }^{22}$ rBMSCs were extracted from the femora of 4-week-old Sprague-Dawley rats, the marrow of the femora and tibia was repeatedly flushed out and finally suspended in h-DMEM (Hyclone) containing 10\% FBS (Hyclone) and 1\% PS (Hyclone) at $37{ }^{\circ} \mathrm{C}$. Cells from 2 to 3 passages were used in this study. All experiments regarding the use of rats were performed in compliance with the National Standards for laboratory animal (GB 14922.1-2001). The use of rBMSCs also followed a protocol approved for this study by the Animal Care and Experiment Committee of Ninth People's Hospital, affiliated to Shanghai Jiao Tong University, School of Medicine.

For cell adhesion observation, cell suspension at the density of $5 \times 10^{4}$ cells per well was seeded on both disks. After incubated for 2 and $24 \mathrm{~h}$, rBMSCs were fixed with $4 \%$ paraformaldehyde at $4{ }^{\circ} \mathrm{C}$ for $30 \mathrm{~min}$. Afterwards, the fixed rBMSCs were treated with $1 \%$ Triton X-100 (Amresco, USA) for 5 min and then washed with PBS twice, followed by staining of cellular cytoskeleton with $5 \mu \mathrm{g} \mathrm{mL^{-1 }}$ phalloidin-iFluor594 conjugate (AAT Bioquest, USA) for $30 \mathrm{~min}$ and further staining of the cellular nuclei with DAPI (Sigma, USA) for $5 \mathrm{~min}$ in darkness. Subsequently, the morphology of rBMSCs on both samples was observed with confocal laser scanning microscope (CLSM) (Leica, Hamburg, Germany). Meanwhile, the expression of integrin $\beta 1$ was detected on two samples. rBMSCs $\left(3.0 \times 10^{4}\right.$ cells per well) were seeded on both disks. After $24 \mathrm{~h}$ of incubation, cells were fixed and permeabilized as mentioned above. After blocked with $3 \%$ BSA for $1 \mathrm{~h}$, anti-integrin $\beta 1$ (Santa cruz, America) was added and incubated overnight at $4{ }^{\circ} \mathrm{C}$. The samples were then incubated with Alexa Fluor® 488 labeled goat anti-rabbit IgG $(\mathrm{H}+\mathrm{L})$ secondary antibody (invitrogen, USA) for 30 minutes at $37^{\circ} \mathrm{C}$. Additionally, the cellular cytoskeleton and the nuclei were stained as above. Finally, the samples were observed using CLSM (Leica, Hamburg, Germany).

Cell Counting Kit-8 (CCK-8, Beyotime Biotech) was used to examine the cell viability of rBMSCs on two disks. Briefly, rBMSCs $\left(4 \times 10^{4}\right.$ cells per well) were cultured on both samples. After 1, 3 and 7 days, the cells were incubated with CCK-8 for $2 \mathrm{~h}$ and microplate reader (Finland) was used to read the optical density of the incubation solution at $450 \mathrm{~nm}$.

\subsection{Osteogenic gene expression assays}

After seeding rBMSCs $\left(3 \times 10^{4}\right.$ cells per well $)$ on two samples for 3 and 10 days, TRIZOL reagent (Invitrogen, USA) was used to isolate total RNA of rBMSCs and PrimeScript 1st Strand cDNA Synthesis kit (TaKaRa, Japan) was used to reverse RNA into complementary DNA (cDNA) as our previous study described. ${ }^{22}$ The expression of RUNX2, ALP, COL1, OPN, OCN, integrin $\alpha 1$ 
and integrin $\beta 1$ was measured by Bio-Rad sequence detection system (MyiQ2, USA). The results were normalized by GAPDH. The primer sets of the above genes were listed in Table 1.

\subsection{Alkaline phosphatase (ALP) activity}

rBMSCs $\left(3 \times 10^{4}\right.$ cells per well) were cultured on both samples for 3 and 10 days. ALP activity at day 3 and 10 was examined according to the instructions of ALP Detection Kit (Beyotime, Shanghai, China) and normalized with total protein. ALP staining at day 10 was performed using BCIP/NBT ALP kit (Beyotime, Shanghai, China). After fixed with $4 \%$ paraformaldehyde and washed with PBS for 3 times, samples were cultured in the mixture of nitro-blue tetrazolium and 5-bromo4-chloro-3-indolyl-phosphate for $30 \mathrm{~min}$. Finally, optical microscopy was used to observe and photograph the samples.

\subsection{Calcium deposition assays}

rBMSCs $\left(2.0 \times 10^{4}\right.$ per well $)$ were seeded on both samples and cultured for up to 14 days. Cells were fixed with $4 \%$ paraformaldehyde for $30 \mathrm{~min}$. After rinsing with PBS for three times, samples were incubated with Alizarin Red S (40 mM) for $15 \mathrm{~min}$. The staining was terminated with PBS and optical microscope was used to observe the samples. Quantitatively, the elution of the staining was acquired by solution of $10 \%$ cetylpyridinium chloride (Sigma) and then measured at $590 \mathrm{~nm}$. Results were normalized with total proteins measured using BCA method.

\subsection{Immunofluorescence of osteogenic proteins}

Initially, rBMSCs $\left(3 \times 10^{4}\right.$ cells per well $)$ were seeded on both samples and cultured for 10 days. The preparation of cells was in accordance with the procedures in cell adhesion assay. Then the samples were incubated with anti-osteopontin (Abcam) and antiosteocalcin (Santa cruz, America) overnight at $4{ }^{\circ} \mathrm{C}$. The samples were then stained with Alexa Fluor ${ }^{\circledR} 488$ labeled goat anti-rabbit IgG $(\mathrm{H}+\mathrm{L})$ secondary antibody (invitrogen, USA) for 30 minutes at $37^{\circ} \mathrm{C}$. After cytoskeleton and nuclei were stained as above, CLSM (Leica, Hamburg, Germany) was used to observe the specimens.

\subsection{Wnt/ $\beta$-catenin pathway-related protein expression}

After seeded on samples for 1 day, the expression of $\beta$-catenin, FZD6, integrin linked ILK and RUNX2 was measured by western blot. The isolation of cellular proteins and the transfer of cell lysates onto nitrocellulose (NC) membranes were conducted as previously described..$^{22}$ The membrane was incubated with anti$\beta$-catenin (1: 2000, Abcam, USA), anti-FZD6 (1: 1000, Abcam, USA), anti- integrin linked ILK (1: 2000, Abcam, USA), antiRUNX2 (1: 1000, Abcam, USA), GAPDH (1: 1000, Abcam, USA) overnight at $4{ }^{\circ} \mathrm{C}$. After rinsed with Tris-buffered saline (TBS) containing $0.05 \%$ Tween-20 (TBST) for 3 times, the membrane was incubated with appropriate horseradish peroxidaseconjugated secondary antibodies for $1 \mathrm{~h}$. Finally, the ECL chemiluminescence reagent (Millipore, USA) was used to develop the immunoreactive bands.

\subsection{Statistical analysis}

All experiments were performed in triplicate and data were expressed as mean \pm standard deviation. Graph Pad Prism software (version 5.0) was used to do data analysis. The skewness and kurtosis test was used to test for normality of distribution of the data. Student's $t$-test was mainly used to analyze the statistical difference between groups. The significant difference was set at a level of $P<0.05$ and the highly significant difference at $P<0.01$.

\section{Results}

\subsection{SEM images of two samples' surface}

The surface characterization of the two samples was shown in Fig. 1. The original SLA surface was covered with macro-pits and micro-pits under lower magnification. However, under a higher magnification, the SLA surface appeared smooth compared with SLA-Ta on the nano-scale (Fig. 1A). While nano-grains were observed in the micron-rough structure of SLA-Ta surface, thus forming a micron/nano hierarchical structure which was showed in Fig. 1B. The cross-sectional view of the SLA-Ta was shown in Fig. 1C. The average thickness of coating Ta film was around $200 \mathrm{~nm}$ and the Ta thin film was tightly bonded to the SLA-Ti base, which was obvious under higher magnification.

\subsection{Surface characterization of two samples}

The surface atomic composition was analyzed using XPS and only characteristic peaks of Ta were detected on the surface of SLA-Ta (Fig. 2A), which indicated the successful implantation of tantalum. As shown in the high-resolution spectra of SLA-Ta (Fig. 2B), the peaks represent the typical binding energy of $\mathrm{Ta}_{2} \mathrm{O}_{5},{ }^{15}$ indicating that Ta on the SLA-Ta surfaces was oxidized

Table 1 Real-time RT-PCR primer sets

\begin{tabular}{lll}
\hline Target gene & Forward primer sequence $\left(5^{\prime}-3^{\prime}\right)$ & Reverse primer sequence $\left(5^{\prime}-3^{\prime}\right)$ \\
\hline GAPDH & GGCAAGTTCAACGGCACAGT & GCCAGTAGACTCCACGACAT \\
RUNX2 & TCTTCCCAAAGCCAGAGCG & TGCCATTCGAGGTGGTCG \\
ALP & GTCCCACAAGAGCCCACAAT & CAACGGCAGAGCCAGGAAT \\
OPN & TGGATGAACCAAGCGTGGA & TCGCCTGACTGTCGATAGCA \\
COL1 & TCTGACTGGAAGAGCGGAGAG & GAGTGGGGAACACACAGGTCT \\
OCN & CAGTAAGGTGGTGAATAGACTCCG & GGTGCCATAGATGCGCTTG \\
Integrin $\alpha 1$ & AGCTGGACATAGTCATCGTC & AGTTGTCATGCGATTCTCCG \\
Integrin $\beta 1$ & AATGTTTCAGTGCAGAGCC & TTGGGATGATGTCGGGAC
\end{tabular}



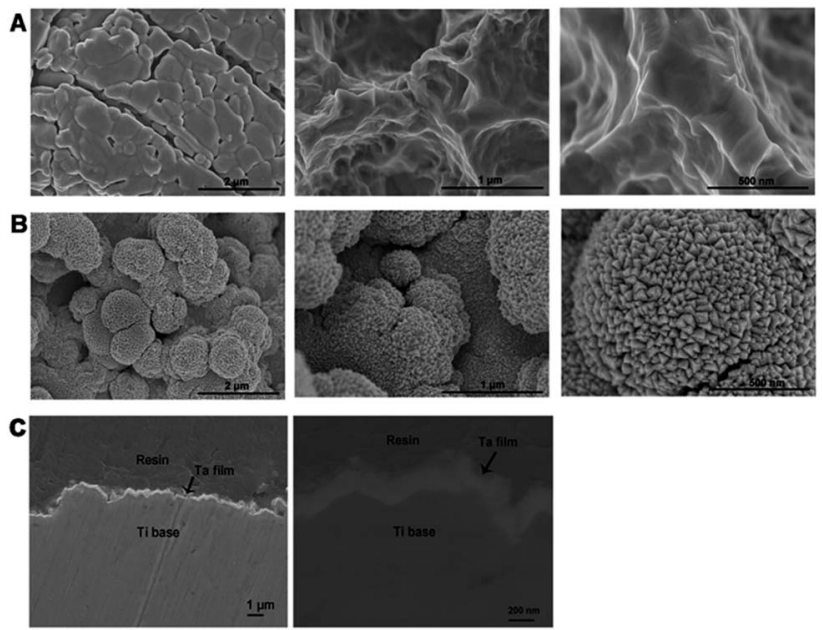

Fig. 1 SEM photographs of the surface morphology of the SLA (A) and SLA-Ta (B) and cross-sectional SEM image of SLA-Ta (C) at various magnifications.
A

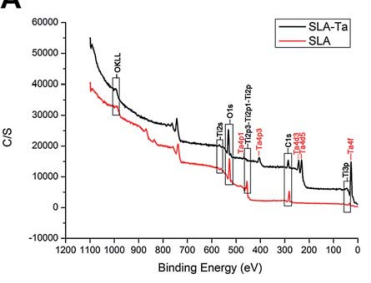

C

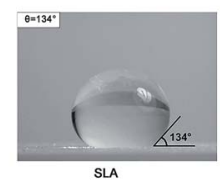

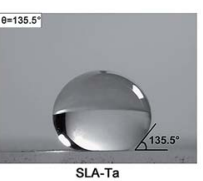

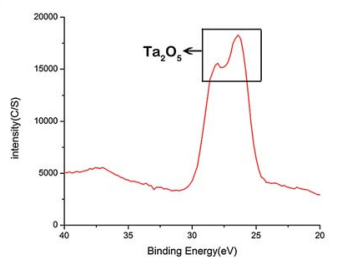

D

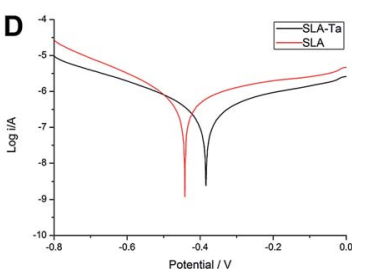

Fig. 2 (A) Full X-ray photoelectron spectra of two surfaces, (B) highresolution XPS spectra of SLA-Ta, (C) water contact angle of two surfaces and (D) potentiodynamic polarization curves of two samples.

and stable. The water contact angle on SLA-Ta was $135.5^{\circ}$, which was comparable to that of SLA, $134^{\circ}$ (Fig. 2C), suggesting that surface modification does not change the surface wettability of titanium. Moreover, Tafel plots of two samples were shown in Fig. 2D and the corrosion potentials (Ecor) of SLA-Ta shift positively compared to SLA, indicating better corrosion resistance formed by the implanted Ta. The surface roughness of the SLA and SLA-Ta were $2.28 \pm 0.11 \mu \mathrm{m}$ and $1.46 \pm 0.09 \mu \mathrm{m}$, respectively (data not shown).

\subsection{Cell adhesion activity of rBMSCs on two surfaces}

Cell adhesion activity of rBMSCs on SLA and SLA-Ta plates were detected at 2 and 24 hours (Fig. 3A). After 2 hours of cell seeding, cells adhering on the SLA-Ta surface was more than that on the SLA group and cells on SLA-Ta also had more filoreticulopodias than that on SLA. However, there was no significance at 24 hours
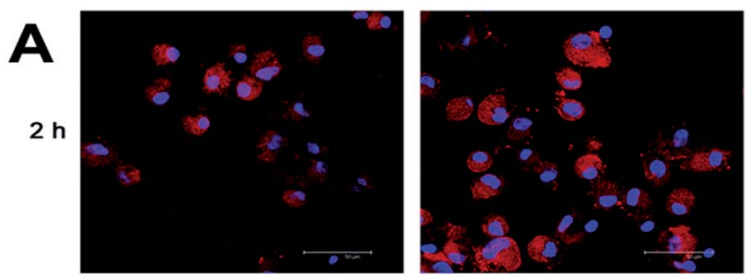

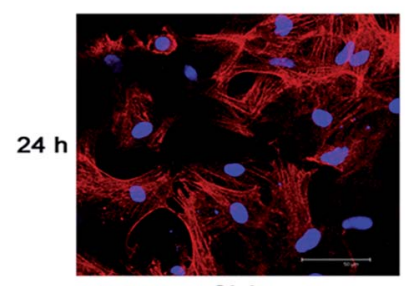

SLA

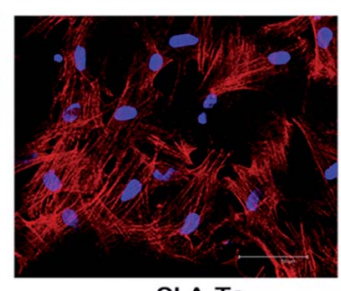

SLA-Ta

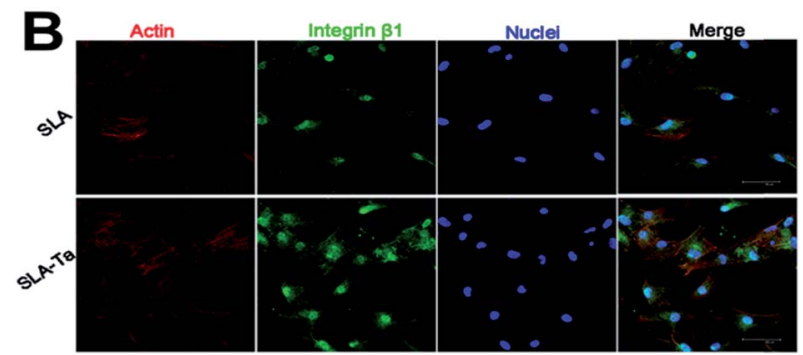

Fig. 3 Cell adhesion assay. (A) Cell nuclei stained with DAPI (blue) and F-actin stained phalloidin-iFlour (red) were imaged by confocal laser scanning microscopy for the two groups at 2 and 24 hours after seeding. (B) Expression of integrin $\beta 1$ at 24 hours was detected by confocal laser scanning microscopy.

between two groups, indicating the excellent early cell adhesion activity of SLA-Ta surface. Additionally, integrin $\beta 1$ protein expression at 24 hours was shown in Fig. 3B and a higher level of integrin $\beta 1$ was expressed on the SLA-Ta surface.

\subsection{Cell viability of rBMSCs on two surfaces}

As shown in Fig. 4, SAL-Ta surface significantly enhanced rBMSCs' viability at day 1, 3 and 7 compared to SLA surface and its cell proliferation rate at day 7 even reached almost $120 \%$.

\subsection{Osteogenic differentiation of rBMSCs on two surfaces}

The osteogenic-related genes expression in rBMSCs cultured on two surfaces was identified by real-time PCR. On SLA-Ta surface, the mRNA level of RUNX-2 in rBMSCs was significantly up regulated at day 3 and 10 (Fig. 5A). The expression of ALP, OPN, COL1 and OCN in rBMSCs was also much higher on SLA-Ta surface compared with SLA surface at day 3 and 10, which suggested SLA-Ta stimulated osteogenic differentiation (Fig. 5E-H). Meanwhile, the expression of cell adhesion-related genes such as integrin $\alpha 1$, integrin $\beta 1$ and fibronectin genes was also detected by PCR assay here, with the results showing that SLA-Ta surface stimulated these genes expression at day 3 (Fig. 5B-D). 


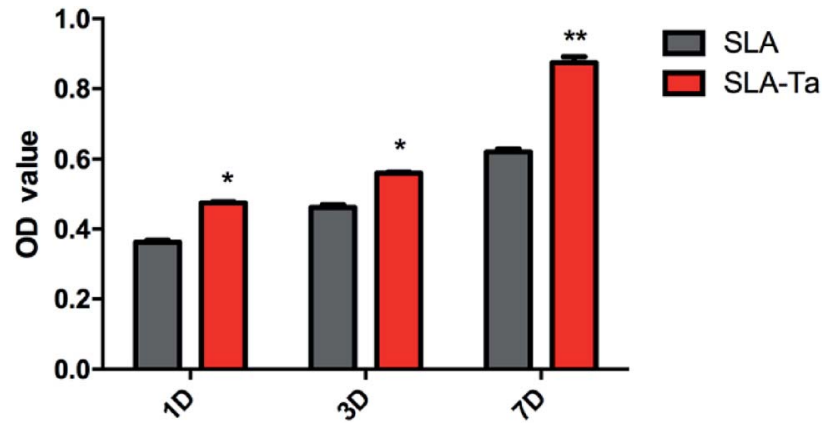

Fig. 4 Cell viability of rBMSCs cultured on SLA and SLA-Ta surfaces for 1,3 and 7 days. Significantly different from SLA group, $* P<0.05, * * P<$ 0.01 .

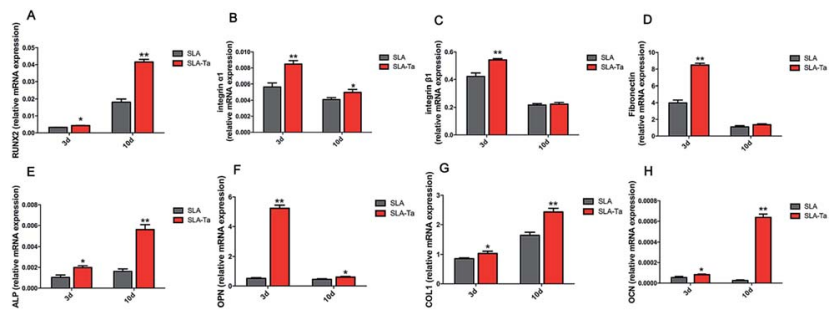

Fig. 5 Gene expression profile of osteogenic differentiation related gene of rBMSCs cultured on two surfaces at day 3 and 10. (A) RUNX2; (B) integrin $\alpha 1$; (C) integrin $\beta 1$; (D) fibronectin; (E) ALP; (F) OPN; (G) COL1; $(H)$ OCN significantly different from SLA group, $* p<0.05$; $* * P<0.01$.

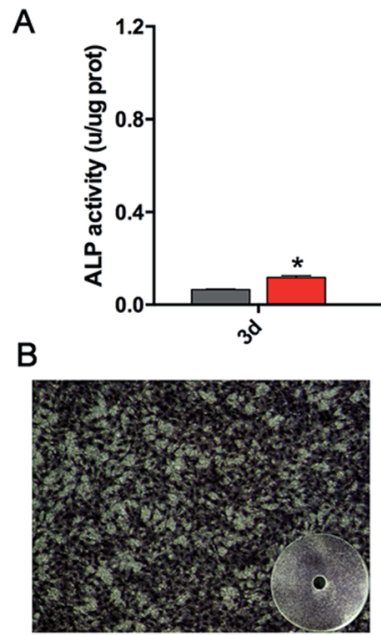

SLA

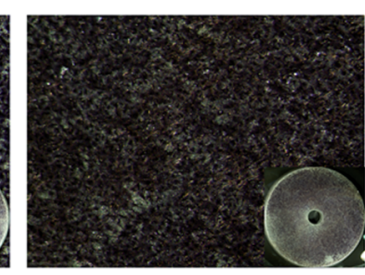

SLA-Ta
Fig. 6 (A) ALP activity expression of rBMSCs seeded on SLA and SLATa surfaces at day 3 and 10, significantly different from SLA group, $* P<$ $0.05, * * P<0.01$, (B) ALP staining of rBMSCs cultured on SLA and SLATa surfaces at day 10 .

\subsection{ALP activity of rBMSCs on two surfaces}

ALP activity in rBMSCs on SLA-Ta was significantly enhanced compared with SLA at day 3 and 10 (Fig. 6A). What's more, ALP staining at day 10 exhibited the same result more directly and

A

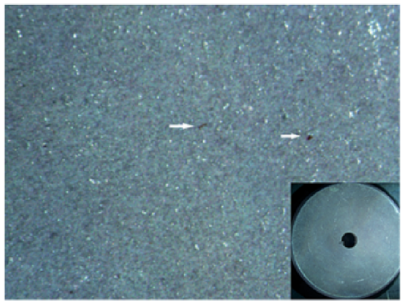

SLA

B

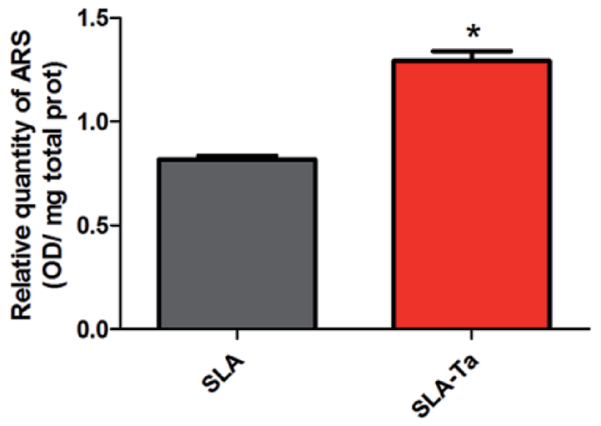

Fig. 7 Calcium deposition assay. After 14 days culture, (A) rBMSCs on SLA and SLA-Ta surfaces were stained with Alizarin Red S solution. (B) Calcium deposition status of cells was assayed using colorimetrically quantitative analysis, significantly different from SLA group, $* P<0.05$. White arrows indicated the bone nodules.

SLA-Ta surface obviously enhanced the ALP activity with the deeper staining (Fig. 6B).

\subsection{Calcium deposition of of rBMSCs on two surfaces}

The bone nodules formation reflecting calcium deposition was measured with the result showing more areas of Alizarin Red S staining for the SLA-Ta surface (Fig. 7A), while little mineralization was found on the SLA surface. According to quantitative analysis, SLA-Ta group also demonstrated significantly higher mineralization ability than that of SLA group (Fig. 7B).

\subsection{OPN and OCN expression of rBMSCs on two surfaces}

The immunofluorescence of OPN/OCN (osteopontin/ osteocalcin) was performed to further confirm osteogenic differentiation activity in rBMSCs on two samples. As showed in Fig. 8, SLA-Ta surface significantly up-regulated OPN and OCN expression with the more areas of green staining.

\subsection{The activation of WNT/ILK- $\beta$-catenin pathway in rBMSCs induced by SLA-Ta surface}

Western blot was used to investigate whether the WNT/ILK- $\beta$ catenin pathway was involved in the osteogenic differentiation of rBMSCs stimulated by SLA-Ta surface. As shown in Fig. 9, the protein expression of $\beta$-catenin, FZD6, integrin-linked ILK and RUNX2 were dramatically increased on SLA-Ta surface compared to SLA group, suggesting that the SLA-Ta surface 

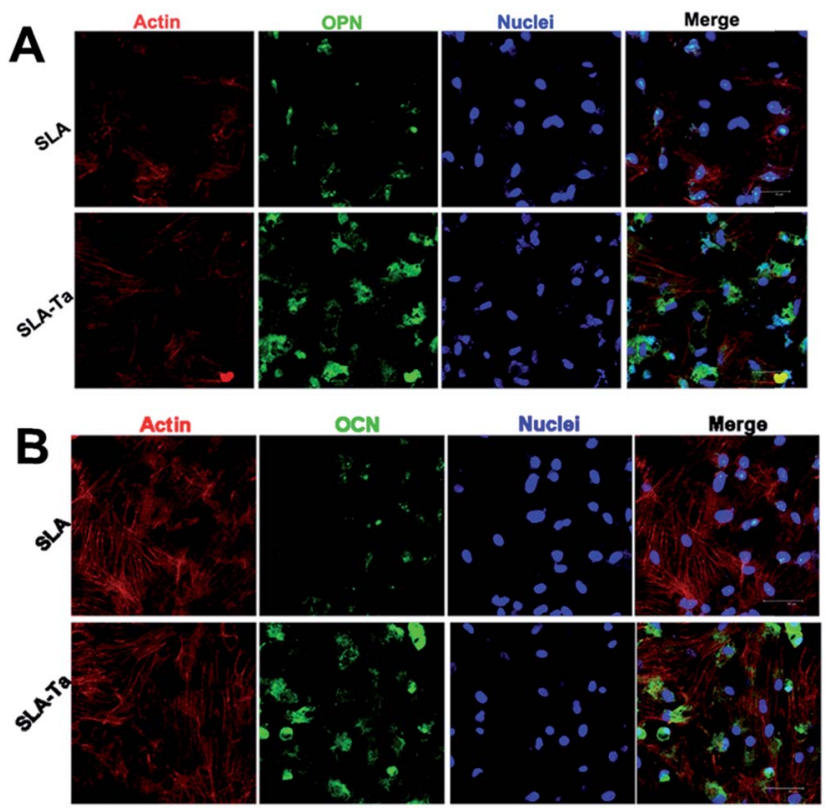

Fig. 8 The expression of OPN (A) and OCN (B) on SLA and SLA-Ta surfaces were detected by immunofluorescence assay at day 10 . "Merge" represents the merged images of actin, OPN/OCN and nuclei.

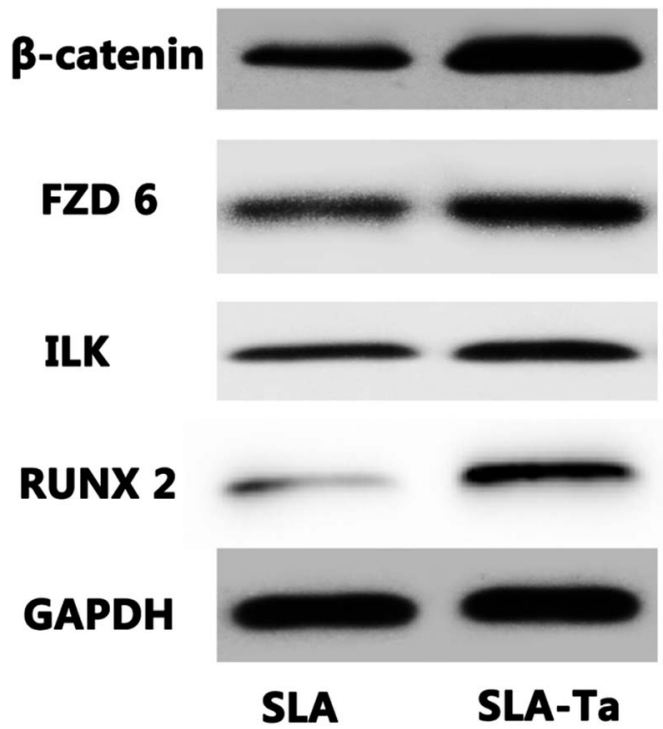

Fig. 9 WNT/ $\beta$-catenin pathway on the osteogenic differentiation of rBMSCs. The expression profile of WNT/ $\beta$-catenin pathway-related proteins in rBMSCs cultured for 1 day on SLA and SLA-Ta surface.

might stimulate the osteogenesis through the activation of WNT/ILK- $\beta$-catenin pathway.

\section{Discussion}

In our study, magnetron sputtering was used to incorporate Ti implant with Ta surface chemistry while maintaining the mechanical advantages of the Ti implant (i.e. relatively low elastic modulus). The aim of this study was mainly to research the osteointegration ability of the fabricated SLA-Ta surface and the underlain mechanism.

Representative macro/nano-structures of the SLA surface modified with Ta were shown in Fig. 1B. Nano grains were superimposed in the micron-rough structure of SLA-Ta surface, thus forming a micron/nano-hierarchical structure and leading to a biological environment conducive to cell attachment and proliferation as well as tissue ingrowth. ${ }^{23}$ As confirmed by XPS (Fig. 2A), the hybrid structure was composed of implanted Ta and $\mathrm{Ti}$ substrate. And the stable $\mathrm{Ta}_{2} \mathrm{O}_{5}$ formed by the implanted Ta not only assured the robustness of the modified surfaces but also significantly increased the binding force between $\mathrm{Ta}$ and $\mathrm{Ti}$ base. ${ }^{15}$ As reported in the previous study, the formation of micro-galvanic couples was detected on the Ag-implanted titanium. ${ }^{21}$ The better corrosion resistance of the new surface (Fig. 2D) might be attributed to the better corrosion resistance of Ta and the micro-galvanic couples formed by the incorporated Ta and Ti base.

It has been proven that the direct interface between an implant and new bone produced by BMSCs forms during the process of osteointegration. In this process, cell adhesion is the initial step, which regulates cell proliferation, migration and differentiation and determines the fate of the biological interfacial interaction with the implant. ${ }^{24}$ In our study, early adhesion of rBMSCs on SLA-Ta surface was superior to that for the control SLA group (Fig. 3A). Cell adhesion has also been proved to occur through binding of integrins, which are receptors that specifically adhere to matrix proteins such as fibronectin and various collagens. ${ }^{23,25}$ In the present study, the expression level of integrin $\alpha 1$, integrin $\beta 1$, and fibronectin genes in rBMSCs seeded on SLA and SLA-Ta substrates were detected (Fig. 5B-D). SLA-Ta surface up regulated these genes expression and also exhibited higher expression of integrin $\beta 1$ protein (Fig. 3B). Several studies have indicated the synergistic effects of hybrid micro/nano-scale structures on osteoblasts

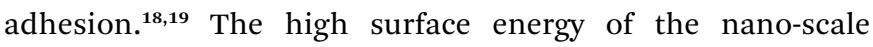
accelerates focal adhesions formation might be one possible explanation. ${ }^{26}$ Previous studies have demonstrated that early load and further deposition of serum proteins on the implant surface, which exist in culture medium, may facilitate initial adhesion of cells. ${ }^{27}$ Another explanation of enhanced cell adhesion by SLA-Ta surface here might be the increased surface areas generated by micro/nano topography modification could maintain the persistence of serum proteins, which could promote the early cell adhesion. In addition, the ability of SLA-Ta surface to promote rBMSCs' viability has also been confirmed (Fig. 4), which might be the synthetic effect of bioactive Ta and the topography that increased initial adhesion of cells.

Surface modification by altering the surface properties such as chemical composition, morphology and mechanical properties et al. is an effective way to tailor specific differentiation of BMSCs. ${ }^{15}$ As aforementioned above, Ta incorporation, hybridstructure formation, and enhanced anticorrosion activity are simultaneously achieved on SLA-Ta surface by surface modification. To evaluate the effect of surface properties on osteointegration of SLA-Ta surface, quantitative measurement of 
osteogenic-related genes expression in rBMSCs was assessed by RT-PCR. In general, rBMSCs on the SLA-Ta surface presented a stronger osteogenic differentiation tendency at genetic level (Fig. 5A and E-H). As we all know, gene transcription is merely the first stage of protein synthesis, increased levels of gene transcription do not necessarily represent the enhanced biological function and the effect is only realized when genes are translated into correspondent proteins. ${ }^{28}$ Therefore, the ALP activity assay, osteopontin/osteocalcin (OPN/OCN) immunofluorescence and calcium deposition were conducted to further confirm osteogenic-stimulated activity of rBMSCs. Fig. 6A showed rBMSCs on Ta-modified surface expressed more ALP activity, and Fig. 6B directly showed more pronounced ALPpositive areas were observed on SLA-Ta surface. As shown in Fig. 8, expression of OPN and OCN, markers related to late osteogenic differentiation, was further detected at the protein level and rBMSCs seeded on the SLA-Ta surface exhibited higher expression of both proteins. SLA-Ta surface also showed more bone nodules than SLA (Fig. 7). Taken together, the results in our study suggested the SLA-Ta surface not only up regulated the osteogenic genes expression but also enhanced the corresponding proteins expression, which reflected the function of osteointegration. In our study, more ALP/OPN/OCN expression and calcium nodes deposition were formed on the SLA-Ta surface. As has been reported, the bioactivity of tantalum is attributed to the formation of Ta-OH. ${ }^{29}$ Besides, $\mathrm{Ta}_{2} \mathrm{O}_{5}$ with nanotopography possess a larger surface area and higher activity to generate Ta-OH groups. ${ }^{15}$ These could be the possible reasons for the excellent osteogenic activity of rBMSCs observed on SLA-Ta surface.

The effect of SLA-Ta surface on the possible signaling pathways of rBMSCs was further studied in an attempt to unveil the hidden molecular mechanism for SLA-Ta to enhance osteointegration. The WNT signalling pathway plays a vital role in various processes such as cell proliferation and differentiation which involved the stabilization of the WNT-Frizzled interac$\operatorname{tion}^{30}$ and the stabilization of $\beta$-catenin in the cytoplasm. ${ }^{31}$ In the present study, the expression of WNT signal-related proteins such as FZD6 and $\beta$-catenin were up regulated by SLA-Ta surface. In addition, integrin linked ILK and RUNX2 expression was also enhanced by SLA-Ta (Fig. 9). Recent study has reported that the signals from topographical cues might activate integrin-linked kinase (ILK)/ $\beta$-catenin pathway to direct osteogenic differentiation of cells. ${ }^{32}$ RUNX-2 protein is a critical transcription factor of osteogenesis and can bind with osteoblast-specific cis-acting element 2, which can further activate the transcriptional activity of downstream osteogenic genes such as ALP, OPN and OCN and correspondent proteins formation. ${ }^{22}$ Specifically, the canonical Wnt pathway could up regulate the osteogenic regulators RUNX2. ${ }^{33}$ Thus, SLA-Ta surface might activate WNT and ILK pathways, both of which intersect on $\beta$-catenin activation to induce RUNX2 expression and then stimulate the downstream osteogenic genes and correspondent proteins formation. However, well-designed studies are further needed to determine the role of these two pathways in SLA-Ta surface's enhanced osteointegration and their correlation.

\section{Conclusions}

The study uses the magnetron sputtering to implant Ta onto SLA-Ti surface to create the SLA-Ta surface with hierarchic micro/nano structure. The corrosion resistance property of SLA$\mathrm{Ti}$ is significantly improved by Ta implantation. The SLA-Ta surfaces also play well in facilitating initial adhesion and spreading, proliferation, differentiation, osteogenic-related genes and proteins expression of rBMSCs. The molecular mechanism of SLA-Ta induces osteointegration might be WNT/ ILK- $\beta$-catenin pathway activation. The enhanced osteogenic activity might be ascribed to the synergistic effects of the implanted bioactive $\mathrm{Ta}_{2} \mathrm{O}_{5}$ and the hybrid micro/nano structure. These findings offered essential theoretical ground for the future application of SLA-Ta implants in orthopedic and dental implantation.

\section{Conflicts of interest}

There are no conflicts to declare.

\section{Acknowledgements}

This work was supported by National Natural Science Foundation of China [81500892, 81470782], Medical-Engineering Cross Fund [YG2015MS10], Shanghai Ninth People's Hospital Fund [Yuan 2015-07] and Shanghai Summit \& Plateau Disciplines.

\section{References}

1 L. Zhao, P. K. Chu, Y. Zhang and Z. Wu, J. Biomed. Mater. Res., Part B, 2009, 91, 470-480.

2 M. Godoy-Gallardo, C. Mas-Moruno, M. C. FernandezCalderon, C. Perez-Giraldo, J. M. Manero, F. Albericio, F. J. Gil and D. Rodriguez, Acta Biomater., 2014, 10, 35223534 .

3 A. Maho, S. Linden, C. Arnould, S. Detriche, J. Delhalle and Z. Mekhalif, J. Colloid Interface Sci., 2012, 371, 150-158.

4 Y. Xie, X. Liu, A. Huang, C. Ding and P. K. Chu, Biomaterials, 2005, 26, 6129-6135.

5 H. Cao, Y. Qiao, X. Liu, T. Lu, T. Cui, F. Meng and P. K. Chu, Acta Biomater., 2013, 9, 5100-5110.

6 V. K. Balla, S. Banerjee, S. Bose and A. Bandyopadhyay, Acta Biomater., 2010, 6, 2329-2334.

7 T. A. Schildhauer, B. Robie, G. Muhr and M. Köller, J. Orthop. Trauma., 2006, 20, 476-484.

8 D. M. Findlay, K. Welldon, G. J. Atkins, D. W. Howie, A. C. Zannettino and D. Bobyn, Biomaterials, 2004, 25, 2215-2227.

9 S. M. Jafari, B. Bender, C. Coyle, J. Parvizi, P. F. Sharkey and W. J. Hozack, Clin. Orthop. Relat. Res., 2010, 468, 459-465.

10 J. Black, Clin. Mater., 1994, 16, 167-173.

11 K. B. Sagomonyants, M. Hakim-Zargar, A. Jhaveri, M. S. Aronow and G. Gronowicz, J. Orthop. Res., 2011, 29, 609-616.

12 J. D. Bobyn, G. J. Stackpool, S. A. Hacking, M. Tanzer and J. J. Krygier, J. Bone Jt. Surg., Br. Vol., 1999, 81, 907-914. 
13 J. D. Bobyn, R. A. Poggie, J. J. Krygier, D. G. Lewallen, A. D. Hanssen, R. J. Lewis, A. S. Unger, T. J. O'Keefe, M. J. Christie, S. Nasser, J. E. Wood, S. D. Stulberg and M. Tanzer, J. Bone Jt. Surg., Am. Vol., 2004, 86(suppl. 2), 123-129.

14 V. K. Balla, S. Bodhak, S. Bose and A. Bandyopadhyay, Acta Biomater., 2010, 6, 3349-3359.

15 T. Lu, J. Wen, S. Qian, H. Cao, C. Ning, X. Pan, X. Jiang, X. Liu and P. K. Chu, Biomaterials, 2015, 51, 173-183.

16 A. Gao, R. Hang, X. Huang, L. Zhao, X. Zhang, L. Wang, B. Tang, S. Ma and P. K. Chu, Biomaterials, 2014, 35, 42234235.

17 C. J. Frandsen, K. S. Brammer, K. Noh, G. Johnston and S. Jin, Mater. Sci. Eng., C, 2014, 37, 332-341.

18 K. C. Popat, L. Leoni, C. A. Grimes and T. A. Desai, Biomaterials, 2007, 28, 3188-3197.

19 R. A. Gittens, T. McLachlan, R. Olivares-Navarrete, Y. Cai, S. Berner, R. Tannenbaum, Z. Schwartz and K. H. Sandhage, Biomaterials, 2011, 32, 3395-3403.

20 Y. Zhu, H. Cao, S. Qiao, M. Wang, Y. Gu, H. Luo, F. Meng, X. Liu and H. Lai, Int. J. Nanomed., 2015, 10, 6659-6674.

21 G. Jin, H. Qin, H. Cao, S. Qian, Y. Zhao, X. Peng, X. Zhang, X. Liu and P. K. Chu, Biomaterials, 2014, 35, 7699-7713.
22 X. Zhang, C. Wu, J. Chang and J. Sun, J. Mater. Chem. B, 2014, 2, 885-891.

23 W. Zhang, Z. Li, Q. Huang, L. Xu, J. Li, Y. Jin, G. Wang, X. Liu and X. Jiang, Int. J. Nanomed., 2013, 8, 257-265.

24 W. Zhang, G. Wang, Y. Liu, X. Zhao, D. Zou, C. Zhu, Y. Jin, Q. Huang, J. Sun, X. Liu, X. Jiang and H. Zreiqat, Biomaterials, 2013, 34, 3184-3195.

25 R. O. Hynes, Cell, 1987, 48, 549-554.

26 A. Diener, B. Nebe, F. Luthen, P. Becker, U. Beck, H. G. Neumann and J. Rychly, Biomaterials, 2005, 26, 383392.

27 G. Mendonca, D. B. Mendonca, F. J. Aragao and L. F. Cooper, Biomaterials, 2008, 29, 3822-3835.

28 A. Leu and J. K. Leach, Pharm. Res., 2008, 25, 1222-1229.

29 T. Kokubo, H. M. Kim and M. Kawashita, Biomaterials, 2003, 24, 2161-2175.

30 L. Schweizer and H. Varmus, BMC Cell Biol., 2003, 4, 4.

31 N. Case and J. Rubin, J. Cell. Biochem., 2010, 110, 545-553.

32 W. Wang, L. Zhao, K. Wu, Q. Ma, S. Mei, P. K. Chu, Q. Wang and Y. Zhang, Biomaterials, 2013, 34, 631-640.

33 C. N. Bennett, K. A. Longo, W. S. Wright, L. J. Suva, T. F. Lane, K. D. Hankenson and O. A. MacDougald, Proc. Natl. Acad. Sci. U. S. A., 2005, 102, 3324-3329. 